\title{
Mechanism of Exfoliation and Prediction of Materials Properties of Clay-Polymer Nanocomposites from Multiscale Modeling
}

\author{
James L. Suter, Derek Groen, ${ }^{\dagger}$ and Peter V. Coveney* \\ Centre for Computational Science, University College London, 20 Gordon Street, London, WC1H 0AJ, United Kingdom
}

Supporting Information

ABSTRACT: We describe the mechanism that leads to full exfoliation and dispersion of organophilic clays when mixed with molten hydrophilic polymers. This process is of fundamental importance for the production of clay-polymer nanocomposites with enhanced materials properties. The chemically specific nature of our multiscale approach allows us to probe how chemistry, in combination with processing conditions, produces such materials properties at the

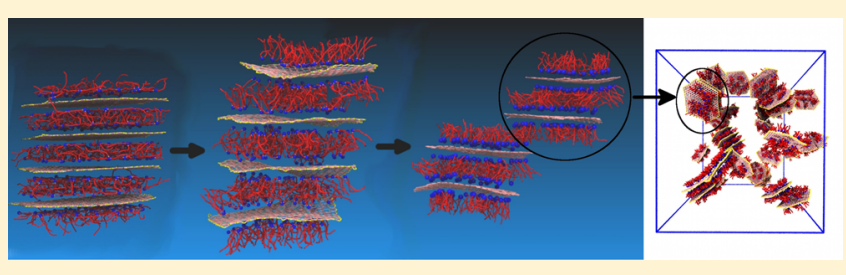
mesoscale and beyond. In general agreement with experimental observations, we find that a higher grafting density of charged quaternary ammonium surfactant ions promotes exfoliation, by a mechanism whereby the clay sheets slide transversally over one another. We can determine the elastic properties of these nanocomposites; exfoliated and partially exfoliated morphologies lead to substantial enhancement of the Young's modulus, as found experimentally.

KEYWORDS: Multiscale modeling, clay-polymer nanocomposites, exfoliation dynamics, materials properties

$\mathrm{T}^{\mathrm{s}}$ he search for new materials with specified performance properties continues apace. The incentives come not only from curiosity driven research but also from the pressure to meet important economic and societal needs. However, the history of innovation in materials design, and the path from initial discovery to implementation in engineering and manufacturing contexts, is a fraught one. Innovations frequently take decades to make their way into commercially viable applications. One of the central reasons for such problems stems from the difficulty of moving between the promise held out at the small ("microscopic") scale by the entity discovered and the behavior of such substances on larger scales appropriate to their use in various applications. This has led to a vast amount of larger scale engineering and manufacturing research and development, almost all of which is conducted experimentally in a largely trial and error fashion. Here we describe a complementary approach, one based on a virtual materials modeling laboratory. Rather than simply synthesizing a great many samples of a material and testing these, we aim to predict materials properties from "first-principles", that is, based on the fundamental description of the starting ingredients in terms of their atomic and molecular composition and structure and the processing conditions.

This is possible through a multiscale scheme that links these very short length (and time) scale descriptions to the larger ones that inform materials properties. We show how our scheme, thus far developed for the study of nanocomposite materials, can be successfully applied to understand and predict the behavior of exfoliating clay-polymer nanocomposites. Within four years of their initial discovery, Toyota was making car components from composites comprised of clay and nylon. However, since that early work, and notwithstanding a vast literature on the subject, the field has failed to live up to expectations. ${ }^{1}$ There remains a very poor level of understanding of the mechanism of action of these materials, and no control over the outcome of mixing arbitrary polymers with clays. ${ }^{2,3}$ Models are therefore required to understand how large-scale dispersion of clay layers in polymers can be achieved and to determine structure-property relationships, thereby enabling the design of materials with desired properties. In this paper, we provide a clear and detailed, chemically specific, explanation for the mechanism of exfoliation of clays by suitable polymers and why, following the dispersion of the individual clay platelets, the resultant materials exhibit very favorable materials properties.

Our multiscale scheme uses short time scale atomistic simulations to create interaction parameters for coarse-grained (CG) particles that represent several atoms. ${ }^{4-6}$ For clay polymer composites, we require a substantial number of atomistic simulations to define all interaction potentials. These simulations include matching to structural parameters using Iterative Boltzmann Inversion ${ }^{5,7}$ and to free-energy profiles for highly interacting CG particles ${ }^{8}$ to build up a complete set of chemically specific CG potentials in the vicinity of clay interfaces. For more details, see Supporting Information. This degree of coarse-graining produces speed-ups of order of 100 times, while retaining molecular specificity, ${ }^{9}$ allowing us to reach length and time scales approaching a hundred nanometers and microseconds, respectively. Almost all atomistic studies of clay-polymer nanocomposites have focused only on the interlayer region between the clay layers, effectively simulating

Received: September 3, 2015

Revised: November 11, 2015

Published: November 17, 2015 
an infinite clay mineral system; such simulations give information on the interfacial behavior at the basal surface but do not describe the large-scale behavior (i.e., dispersion, aggregation) of clay layers. ${ }^{10,11}$ Simulations without molecular detail, such as self-consistent phase field theory, provide predictions about the thermodynamic tendency for clay layers to exfoliate based on a mean field approximation ${ }^{12-17}$ but, again, they cannot describe the chemically specific nature of the clays and polymers. ${ }^{18}$ For a comprehensive review of multiscale modeling of polymer nanocomposites, see Zeng et al. ${ }^{19}$

In a significant advance on our previous multiscale modeling of pristine clays with hydrophilic polymers, which led to intercalated clay-polymer systems, ${ }^{4}$ in this study we examine the process of clay layer exfoliation using similar methods.

To do so, we have chosen a clay, surfactant, and polymer combination that is known to produce at least partially exfoliated clay layers: montmorillonite clay, a quaternary ammonium dimethyldioctadecylammonium ionic surfactant, which has two alkyl chains (each of length $\mathrm{C}_{18}$ ), and hydrophilic poly(ethylene)-glycol (PEG) as the polymer matrix (Figure 1). ${ }^{20-23}$ This combination has been shown exper-

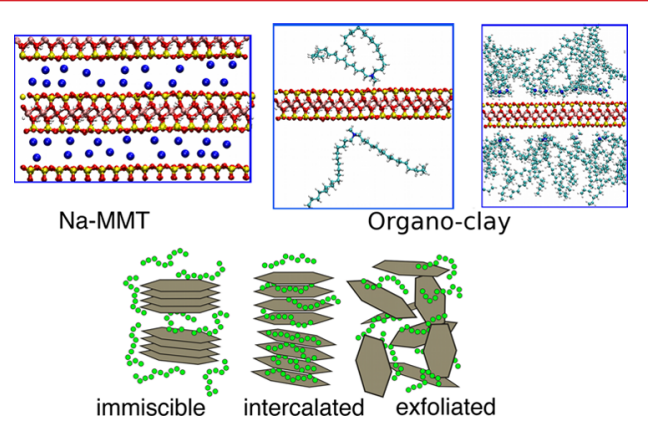

Figure 1. Top left: Na-montmorillonite is a layered mineral consisting of octahedrally coordinated aluminum oxide between two silica tetrahedral layers (the atoms are colored as followed: $\mathrm{Al}=$ pink, $\mathrm{Ai}=$ yellow, $\mathrm{O}=$ red, $\mathrm{H}=$ white, $\mathrm{Na}=$ blue, $\mathrm{C}=$ Cyan). Top right: exchanging the alkali ions in the interlayer for quaternary ammonium ions expands the clay interlayer spacing and gives the clay a hydrophobic character. Bottom: The clay layers can either remain aggregated (immiscible), or can penetrate the clay interlayer (intercalated), or the clay layers can fully disperse (exfoliated).

imentally to produce exfoliated clay layers, as demonstrated by the lack of peaks in X-ray diffractograms following melt intercalation. $^{24}$ Although PEG-clay nanocomposites are often prepared using solution methods, melt processing is a more industrially convenient route of preparation; our simulations therefore take place at elevated temperatures and pressures ( $500 \mathrm{~K}$ and $300 \mathrm{~atm}$ ) in the absence of water.

We have simulated three models of differing clay surface charge density (through isomorphic substitution) and hence surface density of quaternary ammonium surfactants. Each model is comprised of 8 tactoids with each tactoid composed of 4 layers (32 layers in total). Each clay layer is of hexagonal shape with a diameter of approximately $100 \AA$; in total, they comprise approximately $2-6 \%$ of the volume of the simulation cell, depending on whether we use the thickness of the clay sheet $(1 \mathrm{~nm})$ or the initial separation between clay layers $(20-$ $28 \AA$ ) as the thickness of the platelets. The models are listed in Table 1. Each clay layer possesses an aspect ratio of approximately 10 .

Montmorillonite clays are often quoted as having an aspect ratio of between 10 and $1000 ;{ }^{25}$ our models are therefore at the lower end of this distribution. Atomic force microscopy and photocorrelation spectroscopy studies indicated that the sizes of Na-montmorillonite clay exhibited a bimodal distribution with wide size distributions. ${ }^{26}$ The average dimensions of the first population were typically $320-400 \mathrm{~nm}$ long/250 nm wide and 200-250 nm long/120 nm wide for natural and synthetic clays, respectively. The population with smaller sizes were, on average, 65 and $50 \mathrm{~nm}$ long and 35 and $25 \mathrm{~nm}$ wide for natural and synthetic clays, respectively. This is of the same order of magnitude as the lateral dimensions of the clay platelets in our simulations $(10 \mathrm{~nm})$, giving us confidence that our models are representative of clay layers found experimentally.

With an aspect ratio of 10 for our models, the basal clay interactions will dominate, as will be the case for clays with higher aspect ratios. Thus, the results we report should also be applicable to much larger clays, such as those seen in the larger size distribution by Cadene et al. $^{26}$

Initially, the tactoids are in their aggregated state with no polymer in the interlayer gallery. We vary the density from a very low charge montmorillonite clay ( $5 \%$ of $\mathrm{Al}^{3+}$ substituted with $\mathrm{Mg}^{2+}$, referred to as $\mathbf{I}$ in the remainder of this paper), via a typical Wyoming montmorillonite ( $11 \%$, referred to as II) to a high charge density clay ( $17 \%$ substitution rate, referred to as III). These correspond to an approximate cation exchange capacity (CEC) of 0.31 ( 0.28 including clay edges) meq/g, 0.69 (0.62 including clay edges) $\mathrm{meq} / \mathrm{g}$, and 1.05 (0.98 including clay edges) $\mathrm{meq} / \mathrm{g}$, respectively.

Our models were created by constructing a clay tactoid comprising four hexagonal layers, each composed of (110), (010), and (001) surfaces, with lateral dimensions measuring approximately $98 \AA$ by $104 \AA$. Surfactant molecules were then added to this four clay-layer tactoid at the atomistic level, and subsequently relaxed using atomistic molecular dynamics at 500 $\mathrm{K}$, leading to the initial $d$-spacings listed in Table 1 . The partial charges on the edges of the clay sheet were determined through quantum mechanical simulations. ${ }^{27}$ This tactoid was subsequently coarse-grained, and 100 monomer PEG polymers, corresponding to a molecular weight of $4372 \mathrm{~g} \mathrm{~mol}^{-1}$, were built according to the protocol described by Suter et al. ${ }^{4}$ The simulations are replicated in a $2 \times 2 \times 2$ array to form an 8 tactoid (32 clay layers) initial model (see Table 1). All simulations were performed at $500 \mathrm{~K}$ and at relatively high pressure $(300 \mathrm{~atm})$. The lattice parameters were allowed to vary under constant pressure and temperature conditions $(N P T)$ for the first $100 \mathrm{ps}$, after which each simulation was run with constant volume (NVT). The simulations were performed using the LAMMPS molecular dynamics code, ${ }^{28,29}$ using a time

Table 1. Organo-Modified Clay: PEG Models Studied in This Paper

$\begin{array}{ccccc}\text { system } & \text { substitution rate } & \text { simulation cell dimensions }(\AA)^{3} & \text { num. of CG atoms/all atoms equivalent } & \text { initial/intercalated } d \text {-spacing } \AA \\ \text { I } & 5 \% & 407 \times 440 \times 448 & 671200 / 4870912 \\ \text { II } & 11 \% & 415 \times 415 \times 449 & 667904 / 4829856 \\ \text { III } & 17 \% & 504 \times 504 \times 602 & 1363264 / 9568096\end{array}$




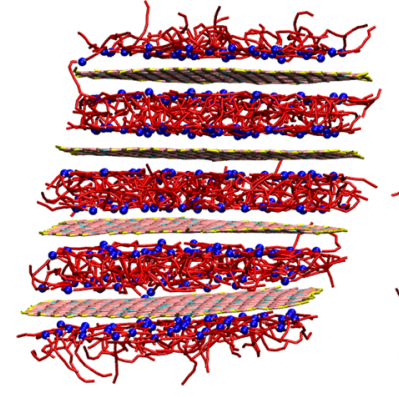

(a)

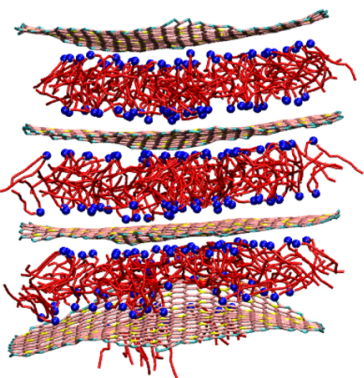

(b)

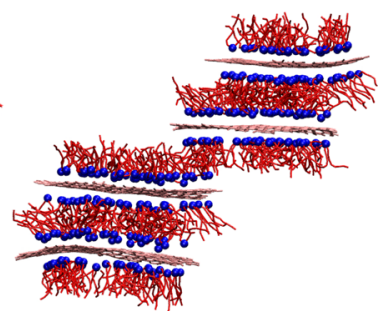

(c)

Figure 2. Snapshots from CG molecular dynamics simulations of a selected alkyl ammonium treated clay tactoid immersed in a melt of PEG polymer. $($ CG clay surface $=$ pink, charge sites $=$ green, edge sites $=$ yellow, surfactant ammonium group $=$ blue, alkyl chains $=$ red. $)$ The PEG polymers have been removed to aid visualization. (a) We show the organoclay model before interaction with the PEG polymer for model II. In (b), the PEG polymer has intercalated into the clay galleries and the separation between the clay layers has increased. In (c), we observe the uppermost two clay layers diffusing away from each other, eventually forming an exfoliated morphology, achieved by the upper clay layers translating in the plane of the clay tactoid layers.

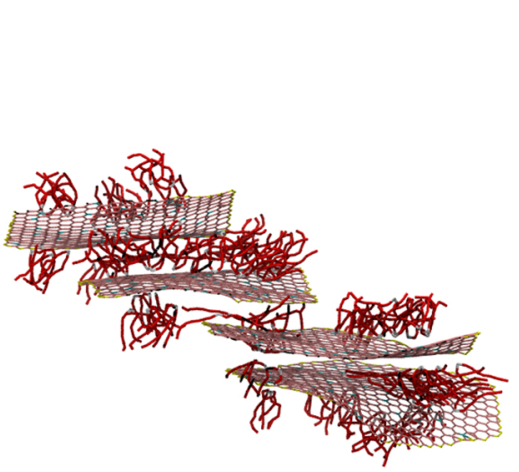

(a)

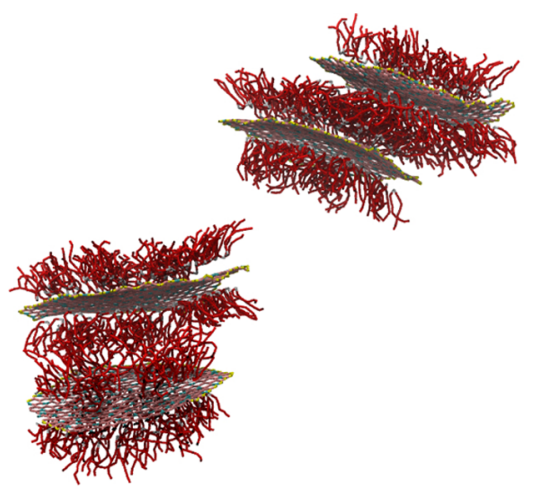

(b)

Figure 3. Snapshots from subdomains of simulations for (a) model I and (b) III after approximately $0.2 \mu$ s. We see that for the low charge system (a), the clay tactoid stack forms a tilted configuration with a large number of alkyl ammonium chains interacting with the bulk PEG polymer (not shown). This configuration appears to be stable on the time scale of our simulations. (b) For model III, the clay layers have already separated and ultimately become fully exfoliated (see Figure 4 c).

step of 2 fs. To facilitate our simulations, we used our pythonbased toolkit, FabMD. ${ }^{30}$ Each simulation was run for $>2 \mu \mathrm{s}$. Further computational details, including the procedure for generating the materials properties, are listed in the Supporting Information.

In all the models listed in Table 1 , we observe an initial expansion as the polymer intercalates between the clay layers over a short time period (approximately $2 \mathrm{~ns}$ ). The intercalated $d$-spacings are shown in Table 1 . Snapshots of this process are shown in Figure 2 for model II. The PEG polymer molecules intercalate into the gallery predominately adjacent to the clay surface, illustrating the favorable interactions between the hydrophilic PEG polymer molecules and the surface as compared to that of the alkyl chains. The rapid dynamics of polymer intercalation has been previously observed experimentally by Vaia and Giannelis and is attributed to the unhindered nature of the confined polymers (there are fewer entanglements, unlike in the bulk).

The separation between the clay layers increases by approximately $7 \AA$. This is consistent with an extra layer of PEG molecules resident on each clay surface. In Supporting Information Figure SI.20, we plot the density profile perpendicular to the clay surface for species within the clay interlayer, which confirms that the majority of the PEG molecules reside on the clay surfaces while the alkyl chains of the surfactant cation extend perpendicular to the clay layers accompanied by clay layer expansion. Once the monolayer of PEG molecules on the clay surface is filled, no further expansion occurs. We can infer that at this point the enthalpic interactions between the polymer and the clay are no longer enough to overcome the entropy loss through polymer confinement. ${ }^{12}$ There is no further driving force for the clay layers to expand. The low density of PEG polymer molecules in the center of the interlayer confirms that the interaction of the PEG polymer with the nonpolar alkyl chains of the surfactant is unfavorable, again lowering the tendency for further PEG polymer intercalation.

For models II and III, once the fast intercalation of PEG molecules has occurred we observe on longer time scales $(>500$ $\mathrm{ns}$ ) the diffusion of the clay layers away from the clay platelets to form exfoliated morphologies. We show this mechanism in Figure 2 for model II and Figure 3 for model III. After more than $3 \mu \mathrm{s}$ of simulation time, the structures of our eight tactoid models are shown for models I, II, and III in Figure 4. We see that for model II there is a mixture of partially and totally exfoliated clay layers while, and for model III all the clay tactoids have exfoliated into single dispersed layers.

We find that the mechanism for the exfoliation of the clay layers does not involve the clay layers expanding perpendicular to the clay surface but rather involves the layers translating 


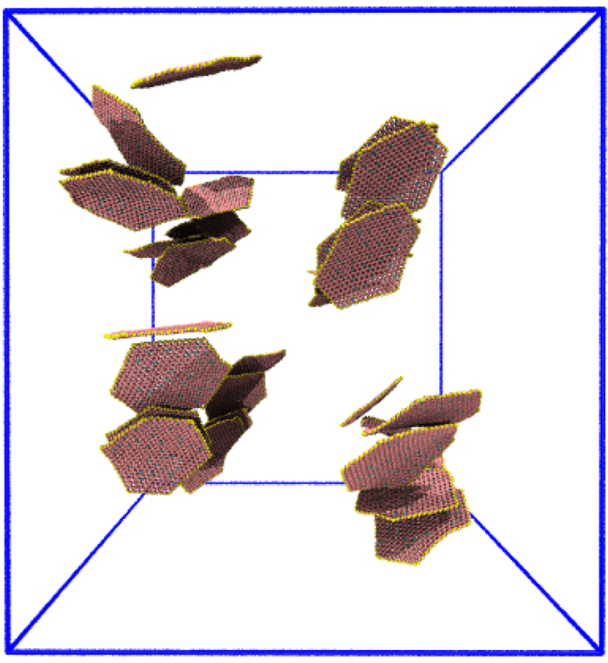

(a)

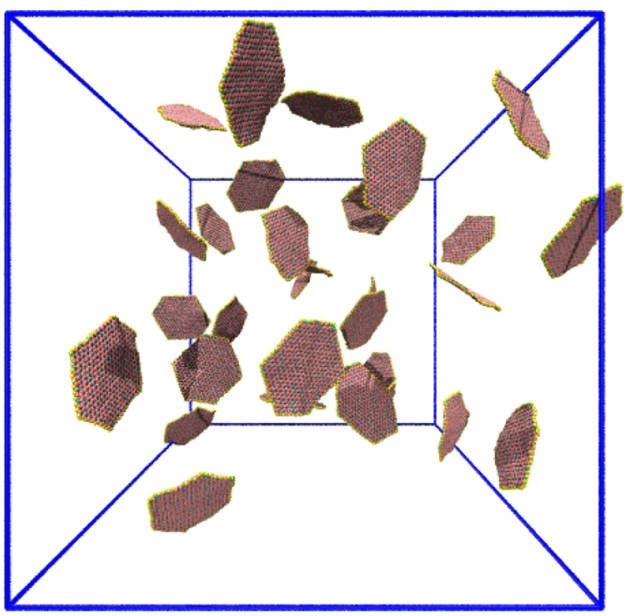

(c)

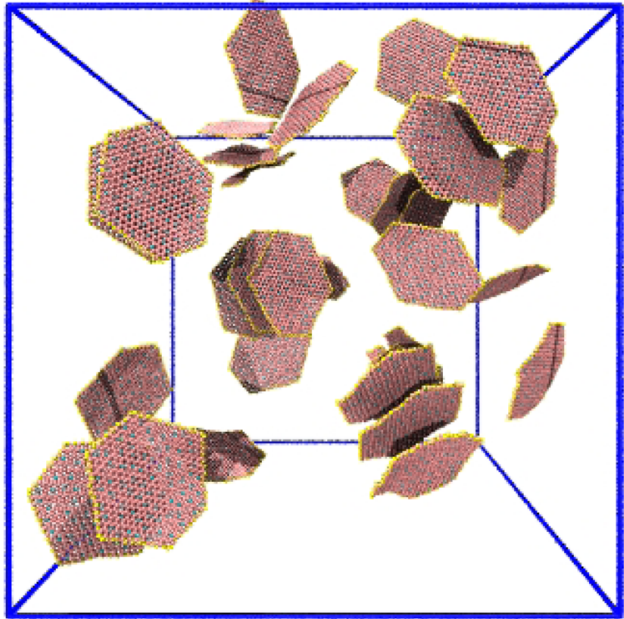

(b)

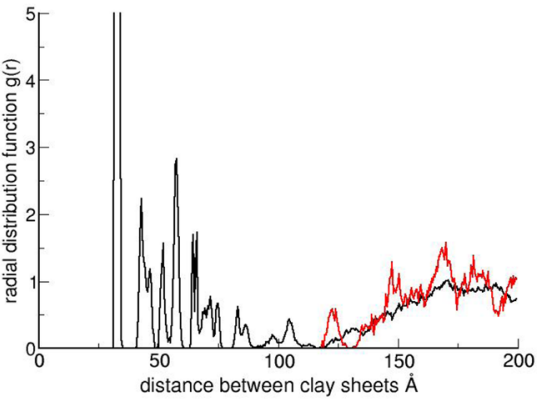

(d)

Figure 4. Snapshots from the CG molecular dynamics simulation, containing 8 tactoids (32 layers) of models (a) I, (b) II, and (c) III after approximately $3 \mu \mathrm{s}$ of simulation. The PEG polymer and surfactant molecules have been removed to aid visualization. (d) The radial distribution function for the center-of-mass of the clay layers for model II (black) and model III (red) averaged over the last 10 ns of simulation, illustrating that model III is fully dispersed.

parallel to one another, as illustrated in Figure 3. For model II, we observe this occurring randomly for different interlayers within the tactoid; for example, in Figure 2 the inner-interlayer exhibits this translational motion, while in other tactoids one of the outer layers undergoes lateral motion and diffuses away.

The incomplete exfoliation of clay layers in model II may be in part due to different sections of the same polymer molecule initially intercalating into either adjacent clay interlayers or bridging between clay layers within the same interlayer. ${ }^{4}$ Where various parts of a single PEG molecule are adsorbed on different clay surfaces, including different interlayers, this serves to resist any translational diffusion of the clay layers (see Figure 5). As a result, for model II we see a distribution of exfoliated and partially exfoliated clay platelets. With much larger platelets, it could be envisaged that the greater number of bridging configurations within the interlayers would resulting in full exfoliation becoming a slower process, leading to a greater number of partially exfoliated platelets. However, for model III, as Supporting Information Figure SI.15 demonstrates, there is much less PEG molecule density in the center of the interlayer, indicating that there are fewer PEG molecules bridging the

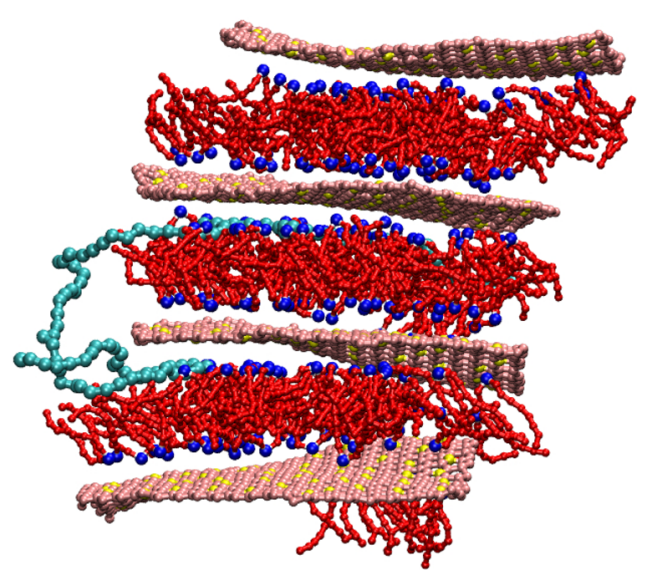

Figure 5. An illustration of a PEG polymer (cyan) adsorbed within two different clay layers in adjacent clay interlayers in model II. Such configurations are likely to inhibit the diffusive process leading to clay layer exfoliation. 
interlayer. This is likely to be due to the combination of a greater number of ammonium groups on the clay surface (restricting the surface area available to the PEG molecules) and the greater initial $d$-spacing. As a result, there is no attraction between the clay layers and they exfoliate rapidly.

Experimentally, we can compare our structural observations with commercially available Cloisite 20A, an organically modified montmorillonite clay with dimethyl dihydrogenated tallow quaternary ammonium surfactants (alkyl chain lengths between $\mathrm{C}_{14}$ and $\mathrm{C}_{18}$ ) and a CEC of 0.95 . In the work of Loyens et al., ${ }^{24}$ when Cloisite 20A was blended with PEG using melt extrusion, a variety of nanostructures were found, depending on polymer molar mass and clay volume fraction: lower molecular mass PEG (100000 $\left.\mathrm{g} \mathrm{mol}^{-1}\right)$ at $5 \%$ clay vol. and small clay volume fractions produced a peak in the smallangle X-ray scattering measurements (SAXS) diffraction spectra at $37 \AA$ as well as at higher spacings. The $32 \AA d$-spacing we have calculated via multiscale modeling for the intercalated structures is therefore in good agreement with experimental observations. In general, intercalation of PEG with molecular weight in the range $10^{5}-10^{6} \mathrm{~g} \mathrm{~mol}^{-1}$ in organically modified clay composites results in reported $d$-spacings in the range of 34 $\pm 4 \AA^{32-36}$ Higher molecular weight PEGs were shown to produce exfoliated morphologies (no peaks in the SAXS diffraction spectra); ${ }^{24}$ we can assume, therefore, that in the lower molecular weight PEG models a combination of exfoliated and intercalated morphologies are present. The Cloisite $15 \mathrm{~A}$ system is a similarly organically modified montmorillonite clay but has a higher CEC of 1.25; this provides a comparison with model III. Although no melt processing has been reported in the literature with PEG polymer, Hyun et al. found that using the solvent casting method the Cloisite 15A nanocomposite showed better dispersion via transmission electron microscopy (TEM) and steady shear viscosity measurements, compared with the Cloisite 20A clay system. ${ }^{33}$

In Figure 6 we show the stress-strain behavior for models II and III, calculated at $100 \mathrm{~K}$, using a strain rate of $1 \times 10^{-8} \mathrm{~s}$. The final snapshots of our simulations were rapidly cooled from 500 to $100 \mathrm{~K}$ over $4 \mathrm{~ns}$ to produce a quenched amorphous system in its glassy state from which stress-strain curves were computed. We have estimated the glass transition temperature

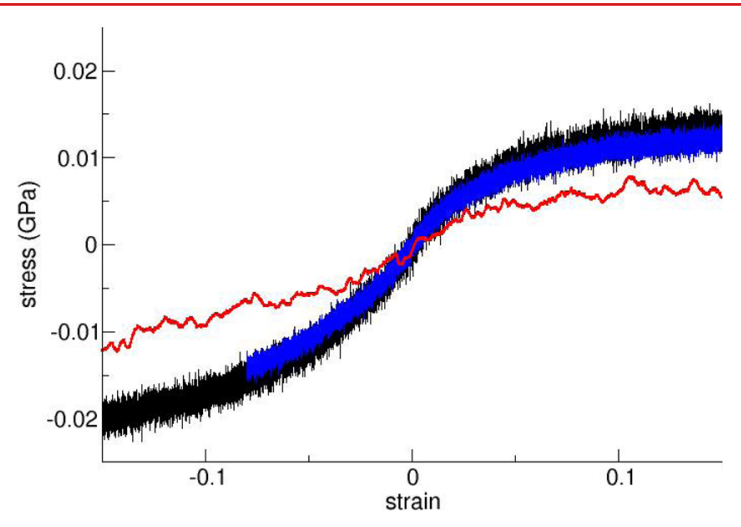

Figure 6. Stress-strain curves for uniaxial compression and extension of our organoclay models II (black) and III (blue). The neat PEG polymer stress-strain curve is also shown (red). We calculate elastic properties (the Young's modulus) from the gradient of the stressstrain curve. The starting configurations for these simulations are the final simulation snapshots shown in Figure 4, cooled down to $100 \mathrm{~K}$.
$\left(T_{\mathrm{g}}\right)$ from the change in the slope of the volume versus temperature curve (see Supporting Information) to be approximately $220 \mathrm{~K}$. We wish to examine the elastic properties in the glassy state of the composite; hence, we have chosen a temperature well below $T_{\mathrm{g}}$ such that we can determine the stiffness, as at this temperature the system will remain in a glassy state when strained. There is no evidence that crystallization of the PEG molecules is occurring (see Supporting Information for more details); we can be sure that we are observing the response of the quenched amorphous system. We find that the stress-strain curve for partially exfoliated model II and totally exfoliated model III are almost identical, even though model III has a smaller clay volume fraction. We find Young's moduli of $0.284 \mathrm{GPa} \pm 0.001$ and $0.266 \mathrm{GPa} \pm 0.001$, respectively; this is over twice that of the neat polymer $(0.126 \mathrm{GPa})$. Our previous models of nonintercalated clay tactoids with a pristine montmorillonite clay immersed in PEG polymer produced a Young's modulus of $0.137 \mathrm{GPa}^{4}$ Note that the calculations reported for the studies at $100 \mathrm{~K}$ are not expected to be quantitatively correct, as they employ potential parametrizations obtained at higher temperatures. However, the qualitative trends are expected to remain intact, as previously found, for example, when examining the Young's modulus of polystyrene-silica composites. ${ }^{37}$

We observe that exfoliated morphologies significantly enhance the elastic properties of the composite and that even partially exfoliated clay morphologies provide a large enhancement. Ratna et al. reported a similar increase in Young's modulus for PEG-clay nanocomposites formed through solution casting, which increased from approximately $0.1 \mathrm{GPa}$ for pure PEG to $0.14 \mathrm{GPa}$ for composites containing $2.5 \%$ clay and to $0.25 \mathrm{GPa}$ for those containing $5 \%$ clay. ${ }^{34}$ The absolute values, although reasonably close, can not be compared due to the qualitative nature of the CG potentials at $100 \mathrm{~K}$ and the solution casting method used experimentally. The enhancement of the Young's modulus of a factor of approximately 2 compared to the bulk polymer is also comparable to a 1.74 factor for the longitudinal stiffness, $E_{11}$, for composites filled with unidirectional disklike particles using the Halpin-Tsai micromechanical model ${ }^{38}$ (see Supporting Information for details).

To summarize, we have shown how organically treated clays promote exfoliation and report the first ever multiscale modeling and simulation study that captures the full process, from the melt intercalation of suitable polymers into such organophilic clays, to the dispersion of individual clay platelets within the polymer matrix. At such longer time scales, we can compute the materials properties of the resultant nanocomposite and find that it exhibits substantially enhanced mechanical properties compared to the properties of its component parts. The predictions of our models are in generally good agreement with experimental observations. In the future, we will investigate the role of processing on materials properties, such as shearing and extensional flow, which are of particular importance in the context of hydrophobic polymers, for example, polystyrene. ${ }^{39}$ Moreover, our multiscale methods are at a very early stage of development and many improvements to their quantitative capabilities can be expected in the future, as well as their transferability between different thermodynamic states. We anticipate that with forthcoming progress in both experimental observations and computational methods the latter will evolve into a powerful tool that will be able to guide materials property prediction 
based on the chemical specificity of the ingredients and their processing conditions.

\section{ASSOCIATED CONTENT}

\section{S Supporting Information}

The Supporting Information is available free of charge on the ACS Publications website at DOI: 10.1021/acs.nanolett.5b03547.

The tabulated coarse-grained potentials used in this study are available at http://ccs.chem.ucl.ac.uk/. Derivation of coarse-grained interaction potentials, discussion of the clay structures used in this study, the intercalated density profiles and the software management toolkit used in this study. (PDF)

\section{AUTHOR INFORMATION}

\section{Corresponding Author}

*E-mail: p.v.coveney@ucl.ac.uk.

\section{Present Address}

${ }^{\dagger}$ Department of Computer Science, Brunel University London, Uxbridge, Middlesex, UB8 3PH, United Kingdom

\section{Notes}

The authors declare no competing financial interest.

\section{ACKNOWLEDGMENTS}

This work was funded in part by the EU FP7 MAPPER project (RI-261507), the EU Horizon 2020 ComPat project (671564), and the Qatar National Research Fund (09-260-1-048). Supercomputing time was provided by PRACE (project PRA044), the Hartree Centre (Daresbury Laboratory), and ARCHER, the U.K. national supercomputing facility at the University of Edinburgh, via EPSRC through Grants EP/ F00521/1, EP/E045111/1, EP/I017763/1, and UK COMES $(\mathrm{EP} / \mathrm{L} 00030 \mathrm{X} / 1)$. Data storage was provided by EUDAT (283304).

\section{REFERENCES}

(1) Okada, A.; Usuki, A. Macromol. Mater. Eng. 2006, 291, 14491476.

(2) Chen, B.; Evans, J. R. G.; Greenwell, H. C.; Boulet, P.; Coveney, P. V.; Bowden, A. A.; Whiting, A. Chem. Soc. Rev. 2008, 37, 568-594.

(3) Ray, S. S. Macromol. Chem. Phys. 2014, 215, 1162-1179.

(4) Suter, J. L.; Groen, D.; Coveney, P. V. Adv. Mater. 2015, 27, 966-984.

(5) Ruehle, V.; Junghans, C.; Lukyanov, A.; Kremer, K.; Andrienko,

D. J. Chem. Theory Comput. 2009, 5, 3211-3223.

(6) Karabasov, S.; Nerukh, D.; Hoekstra, A. G.; Chopard, B.; Coveney, P. V. Philos. Trans. R. Soc., A 2014, 372, 20130390.

(7) Müller-Plathe, F. ChemPhysChem 2002, 3, 754-769.

(8) Roux, B. Comput. Phys. Commun. 1995, 91, 275-282.

(9) Johnston, K.; Harmandaris, V. Soft Matter 2013, 9, 6696-6710.

(10) Cygan, R. T.; Greathouse, J. A.; Heinz, H.; Kalinichev, A. G. J. Mater. Chem. 2009, 19, 2470-2481.

(11) Greathouse, J. A.; Johnson, K. L.; Greenwell, H. C. Minerals 2014, 4, 519-540.

(12) Vaia, R. A.; Giannelis, E. P. Macromolecules 1997, 30, 79907999.

(13) Balazs, A. C.; Singh, C.; Zhulina, E. Macromolecules 1998, 31, 8370-8381.

(14) Buxton, G. A.; Balazs, A. C. J. Chem. Phys. 2002, 117, 76497658

(15) Balazs, A. C.; Bicerano, J.; Ginzburg, V. V. In Polyolefin Composites; Nwabunma, D., Kyu, T., Eds.; John Wiley \& Sons, Inc.: Hoboken, NJ, 2008; pp 415-448.
(16) Ginzburg, V. V.; Balazs, A. C. Macromolecules 1999, 32, 56815688.

(17) Ginzburg, V. V.; Singh, C.; Balazs, A. C. Macromolecules 2000, 33, 1089-1099.

(18) Ginzburg, V. V.; Weinhold, J. D.; Jog, P. K.; Srivastava, R. Macromolecules 2009, 42, 9089-9095.

(19) Zeng, Q.; Yu, A.; Lu, G. Prog. Polym. Sci. 2008, 33, 191-269.

(20) Heinz, H.; Koerner, H.; Anderson, K. L.; Vaia, R. A.; Farmer, B. L. Chem. Mater. 2005, 17, 5658-5669.

(21) Fu, Y.-T.; Heinz, H. Chem. Mater. 2010, 22, 1595-1605.

(22) Liu, X.; Lu, X.; Wang, R.; Zhou, H.; Xu, S. Clays Clay Miner. 2007, 55, 554-564.

(23) Losego, M. D.; Blitz, I. P.; Vaia, R. A.; Cahill, D. G.; Braun, P. V. Nano Lett. 2013, 13, 2215-2219.

(24) Loyens, W.; Jannasch, P.; Maurer, F. H. Polymer 2005, 46, $903-$ 914.

(25) Ray, S. S.; Okamoto, K.; Okamoto, M. J. Appl. Polym. Sci. 2006, $102,777-785$.

(26) Cadene, A.; Durand-Vidal, S.; Turq, P.; Brendle, J. J. Colloid Interface Sci. 2005, 285, 719-730.

(27) Suter, J. L.; Kabalan, L.; Khader, M.; Coveney, P. V. Geochim. Cosmochim. Acta 2015, 169, 17-29.

(28) Plimpton, S. Large-scale atomic/molecular massively parallel simulator. http://lammps.sandia.gov (accessed Nov. 18, 2015)

(29) Plimpton, S. J. J. Comput. Phys. 1995, 117, 1-19.

(30) Groen, D.; Bhati, A.; Suter, J. L.; Hetherington, J.; Zasada, S. J.; Coveney, P. V. FabMD. https://github.com/UCL-CCS/FabSim, 2015 (accessed Nov. 18, 2015).

(31) Krishnamoorti, R.; Vaia, R. A.; Giannelis, E. P. Chem. Mater. 1996, 8, 1728-1734.

(32) Kelarakis, A.; Giannelis, E. P. Polymer 2011, 52, 2221-2227.

(33) Hyun, Y. H.; Lim, S. T.; Choi, H. J.; Jhon, M. S. Macromolecules 2001, 34, 8084-8093.

(34) Ratna, D.; Divekar, S.; Samui, A.; Chakraborty, B.; Banthia, A. Polymer 2006, 47, 4068-4074.

(35) Choi, H. J.; Kim, S. G.; Hyun, Y. H.; Jhon, M. S. Macromol. Rapid Commun. 2001, 22, 320-325.

(36) Lim, S.; Kim, J.; Chin, I.; Kwon, Y.; Choi, H. Chem. Mater. 2002, 14, 1989-1994.

(37) Rahimi, M.; Iriarte-Carretero, I.; Ghanbari, A.; Böhm, M. C.; Müller-Plathe, F. Nanotechnology 2012, 23, 305702.

(38) Sheng, N.; Boyce, M. C.; Parks, D. M.; Rutledge, G.; Abes, J.; Cohen, R. Polymer 2004, 45, 487-506.

(39) Bujdak, J. J. Phys. Chem. C 2015, 119, 12016-12022. 